\title{
A Review of the Fundamental Design Problems of Providing Ambulation for Paraplegic Patients
}

\author{
J. Stallard, BTech, CEng, MIMechE, R. E. Major, BSc, CertEd, J. H. \\ Patrick, FRCS \\ The Orthotic Research E Locomotor Assessment Unit, The Robert fones E Agnes \\ Hunt Orthopaedic Hospital, Oswestry, Shropshire, SY10 7AG, UK.
}

\begin{abstract}
Summary
Walking for paraplegics with complete mid-thoracic lesions is now becoming a clinically viable reality. However, much of the literature in this field overstates the achievements and masks the difficulties that patients may encounter.

This paper highlights Independence, Energy Cost, Cosmesis, Reliability and Cost as the five areas of prime interest involved in the design compromise for any walking system and urges that all relevant information for any system should be readily available to prescribers. Currently available walking systems are briefly considered.

Key words: Paraplegia; FES (Functional Electrical Stimulation); Orthosis; Walking.
\end{abstract}

Much publicity has been given to the achievement of reciprocal walking for paraplegic patients with complete lesions at Ll or above in both the popular media and in medical publications. The more sensational reports have implied that these patients can now be offered practical systems which enable them to achieve almost normal walking function. In truth the currently available clinically viable options involving mechanical orthoses fall far short of that objective and the systems involving pure electrical stimulation of paralysed muscles are not yet even able to match the performance of the better mechanical systems. Nevertheless important advances have been achieved and paraplegic patients with complete thoracic lesions up to T4 for instance, can now walk using a reciprocal mode with an efficiency similar to that previously demonstrated by patients with lumbar lesions using swing through gait. It is essential that a sense of proportion be kept by those responsible for prescription if these new advances are not to be damaged by a wave of disappointment when they fail to live up to unrealistic expectations. Clinicians will be unable to do this unless designers and commercial suppliers of apparatus provide them with the relevant data relating to all aspects of a particular treatment system.

There are a number of fundamental requirements which any walking system 
must satisfy and in examining a particular option their effectiveness in providing each of these should be assessed. Inevitably a number of design compromises will have been made by those who develop a system. If optimum treatment for every patient is to be achieved prescribers need to be aware of both the fundamental requirements and the compromises which particular systems have made and to compare these with the needs and desires of the patients. In discussing the various approaches for the provision of ambulation for paraplegics, an orthosis is defined as a device which assists limb function and therefore includes both mechanical and electrical designs.

\section{Fundamental requirements for walking}

Any form of walking requires (Rose, 1980):

1. that the multi-segmented structure of the body be stabilised to prevent it collapsing or toppling.

2. the injection of propulsive forces.

3. control of the forces which provide stability and propulsion.

Thoracic lesion patients are unable voluntarily to control their lower limbs to provide these functions so that mechanical stabilisation by three point fixation of the joints (Rose, 1986) or electrical stimulation of relevant muscles must be used (Petrofsky and Phillips, 1983). As regards propulsion, this must be achieved though the upper limbs via walking frames or (preferably) crutches for any of the systems currently envisaged, though supplementary inputs by electrical stimulation of the lower limbs are used in systems employing functional electrical stimulation (FES) (Andrews and Bajd, 1985; Patrick and McClelland, 1985).

Reciprocal walking in which one leg is sequentially placed in front of the other has three additional requirements to those for swing through gait. These are:

4. Clearing the foot of the swing leg whilst the stance leg continues to provide a large share of the weight bearing function.

5. Forward swing of the swing leg.

6. Forward progression of the trunk over the stance leg.

Paraplegic patients require orthoses and/or FES in order to achieve each of these.

\section{Determinants of the design compromise}

Although walking is the prime function required there are other factors involved which will affect the suitability of the solution for any particular patient. By considering these parameters, clinicians will better be able to select a device to match patient needs. These additional factors are:

\section{Independence}

To be of real value, walking for a paraplegic patient should be achieved completely independently. This means that the patient must be able to put on and 
take off the complete system, transfer from sitting to standing and vice-versa, without any assistance from a helper. Additionally the walking should include the ability to tackle gentle slopes and single small steps such as kerbs. The ability to negotiate a flight of steps is desirable, but rarely demonstrated by patients with mid thoracic lesions.

Other aspects of independence include problems of toiletting, if it is intended that it should be worn throughout the day, and the means of achieving additional activities (other than walking), when wearing the orthosis.

\section{Energy cost}

One of the main benefits expected of a reciprocal walking system is a reduction in energy cost, because it eliminates the need to raise the whole of body weight via the upper limbs for each stride, as in swing through gait. The degree to which this saving occurs will depend on the effectiveness of the system used in permitting the swing leg to be cleared from the ground and the means of achieving forward progression of the trunk. In examining the suitability of a system for a particular patient, attention should be paid to methods employed to achieve these factors, the patient's ability to generate the necessary forces and the published results of energy costs of the system.

\section{Cosmesis}

Because cosmesis is such an emotive subject it is often not considered in a suitably objective manner. Until total normality is achieved, cosmesis will be a compromise of a number of different factors, all of which are very important. Those which are most important will depend on an individual patients own attitudes. The main factors concerned in cosmesis are:

The style of walking, the aim being that it should resemble normal walking as closely as possible.

The ability to disguise the wearing of the orthosis, the ease with which clothes can cover the device being the important factor.

The clumsiness of the walking aid used in conjuction with the orthosis. At present all systems use walking aids, but only a few are able to achieve effective walking with crutches.

The aesthetic design of the orthosis and other apparatus used by the patient.

\section{System reliability}

For a system to be truly practical it must have a high level of reliability so that its user is not frequently deprived of the walking function. It should also be designed so that any failures which occur do not put the patient at risk of physical injury. Any mechanical system will have to sustain very high loadings if it is to operate efficiently for the patient and will need careful design to avoid failure. Pure FES systems will have to address the problem of achieving a safe mode of failure, which mechanical systems can achieve with appropriate detail design, so that patients do not collapse when a malfunction occurs. 


\section{System cost}

In the current financial climate it has become ever more important for clinicians to consider the cost of the treatments which they prescribe. There are no cheap options for systems which enable the paraplegic to walk, and the overall cost is made up of a number of different factors, many of which are frequently ignored. The main elements of treatment cost are:

The primary apparatus (orthosis, FES system etc)

The Walking Aids required

Surgery required to enable orthotic fit and function

Surgery implicit in the design of treatment

Fitting and Training (These involve Clinical Staff)

Maintenance, including routine replacement, repairs and on-going patient assessment.

\section{Currently available systems}

There are three fundamental approaches to the provision of reciprocal walking for high level paraplegic patients:

1. Purely Mechanical Orthoses.

2. Hybrid devices comprising mechanical orthosis with supplementary FES.

3. Purely FES system with no mechanical orthosis.

None of these systems envisages walking without the use of additional walking aids.

At the present time only the first of these approaches can provide treatment in a truly clinical context. There are at least two modern mechanical orthoses which are available routinely on an international basis, the Louisiana State University Reciprocating Gait Orthosis (Douglas et al., 1983; Beckman, 1987) and the ORLAU ParaWalker (Stallard et al., 1986; Butler and Major, 1987). Both of these have attempted to provide all of the fundamental requirements of walking for paraplegic patients. The developers of both have recognised the need for compromise in design and although they are ostensibly similar, there are differences in the solutions adopted and consequently the degree to which each of the specific requirements is achieved. Because compromises are made the requirements of patients need to be considered very carefully by the clinician before the decision is taken as to which system addresses their requirements most appropriately. As further data is published beyond that which already exists (Nene and Patrick, 1987; Merritt, 1987), this can also be examined in relation to all the fundamental requirements.

The hybrid and pure FES approaches to paraplegic walking are both being extensively researched. It has already been established that a hybrid approach can further improve the efficiency of walking, despite the relative crudeness of the stimulation parameters (McClelland et al., 1987). A number of practical problems relating to the siting and location of electrodes, the design of suitable production stimulators and control systems, and the training regimes for patients remain to be solved before a routine clinical treatment can be made available. That this can be achieved now seems to be a viable proposition. However, such 
a solution involves further design compromises and these too must be considered when decisions on treatment are made. Hybrid systems will undoubtedly have a higher initial cost, are likely to have greater routine maintenance costs and lower system reliability, and will present different cosmetic problems.

Much exciting work is being done in the development of pure FES systems (Bajd et al., 1983; Kralj and Grobelnik, 1973; Marsolais and Kobetic, 1986; Vossius, 1985). Whilst such a system would be the ideal solution, there are many difficulties to be resolved before all of the fundamental problems can be overcome. In particular, system reliability, energy cost, safe failure mode for patients, cosmesis of walking and cost are all areas in which pure FES systems can not yet match the mechanical or hybrid approach. Clearly modern technology could provide the solutions of these deficiencies and the excellent work being carried out on an international scale needs every encouragement so that this option can be made clinically viable as soon as possible.

\section{Conclusion}

Reciprocal walking for paraplegic patients with complete thoracic lesions is now a viable clinical option. At present only mechanical systems are routinely available and the compromises which they have to make mean that walking is limited to specific groups of patients. Research into hybrid and pure FES systems is continuing and as they become available they will inevitably have evolved new compromises.

It is unlikely that any one system will provide the ideal solution for every patient, and clinicians will need to be able to assess which fundamental approach will best suit particular groups of patients. Children, adolescents, young and old patients all present different problems requiring different approaches and subdivision of needs within these groups will probably occur.

Before any system can claim to be a routine clinical treatment it must address and answer all of the problems created by the fundamental requirements. This inevitably means that designers and developers will have to make compromises and it is incumbent upon them to make clear to clinicians what those compromises are and the means by which they provide the five fundamental requirements.

It is essential that anyone who prescribes walking systems should understand the five fundamental requirements so that they can establish the degree to which any system meets the needs of their patients.

\section{References}

ANDREws BJ, BAJd T 1985 Paraplegic locomotions: hybrid FES approach. Conference on Medical and Biological Engineering and Medical Physics. ESPOO, Finland 11-16 August.

BAjD T, KRALJ A, TURK R, et al. 1983 The use of a four-channel electrical stimulator as an ambulatory aid for paraplegic patients. Physical Therapy 63(7):1116-1120.

BeCKMANn J 1987 The Louisiana State University Reciprocating Gait Orthosis. Physiotherapy 73(8):386-392.

Butler PB, MAJOR RE 1987 The ParaWalker: A rational approach to the provision of reciprocal ambulation for paraplegic patients. Physiotherapy 73(8):393-397.

Kralj A, Grobelnik S 1973 Functional stimulation-A new hope for paraplegic patients? Bulletin of Prosthetic Research Fall:75-102. 
MARSOLAIS EB, KoBETIC MS 1986 Implantation techniques and experience with percutaneous intramuscular electrodes in the lower extremities. Fournal of Rehabilitation in Research and Development 23(3):1-8.

McClelland M, ANdrews BJ, Patrick JH, et al. 1987 Augmentation of the Oswestry ParaWalker orthosis by means of surface electrical stimulation: Gait analysis of three patients. Paraplegia 25:32-38.

MERRITT JL 1987 Knee-ankle-foot orthosis, long leg braces and their practical applications. Physical Medicine and Rehbailitation: State of the Art Review 1(1):67-82.

Nene A, PATRICK JH 1986 Energy cost of paraplegic locomotion with the ORLAU ParaWalker. Paper read at the Scientific Meeting International Medical Society of Paraplegia, Stoke Mandeville, May.

Patrick JH, MCClelland MR 1985 Low energy reciprocal walking for the adult paraplegic. Paraplegia 23:113-117.

Petrofsky JS, Phillips CA 1983 Computer controlled walking in the paralysed individual. fournal of Neurological and Orthopaedic Medicine and Surgery 4(2):153-164.

Petrofski JS, Phillips CA, Larson P, et al. 1985 Computer synthesised walking: An application of orthosis and functional electrical stimulation (FES). Fournal of Neurological and Orthopaedic Medicine and Surgery 6(3):219-230.

RosE GK 1980 Orthoses for the severely handicapped-rational or empirical choice. Physiotherapy 66:76-81.

RosE GK 1986 Orthotics: Principles and practice. William Heinemann Medical Books London, pp. 47-48.

Stallard J, Major RE, Poiner R, et al. 1986 Engineering design considerations of the ORLAU ParaWalker and FES hybrid system. Engineering in Medicine 15(3):123-129.

Vossius G 1985 Common aspects of functional electrical stimulation. Proceedings of the EEC COMAC/BME Topical Workshop 'Restoration of Walking for Paralysed Persons', pp 27-28. 